\title{
In vivo Motion Correction in Super Resolution Imaging of Rat Kidneys
}

Taghavi, Iman; Bech Andersen, Sofie ; Hoyos, Carlos A. Villagomez ; Nielsen, Michael Bachmann; Sørensen, Charlotte Mehlin; Jensen, Jørgen Arendt

Published in:

IEEE Transactions on Ultrasonics, Ferroelectrics and Frequency Control

Link to article, DOI:

10.1109/TUFFC.2021.3086983

Publication date:

2021

Document Version

Peer reviewed version

Link back to DTU Orbit

Citation (APA):

Taghavi, I., Bech Andersen, S., Hoyos, C. A. V., Nielsen, M. B., Sørensen, C. M., \& Jensen, J. A. (2021). In vivo Motion Correction in Super Resolution Imaging of Rat Kidneys. IEEE Transactions on Ultrasonics, Ferroelectrics and Frequency Control, 68(10), 3082 - 3093. https://doi.org/10.1109/TUFFC.2021.3086983

\section{General rights}

Copyright and moral rights for the publications made accessible in the public portal are retained by the authors and/or other copyright owners and it is a condition of accessing publications that users recognise and abide by the legal requirements associated with these rights.

- Users may download and print one copy of any publication from the public portal for the purpose of private study or research.

- You may not further distribute the material or use it for any profit-making activity or commercial gain

- You may freely distribute the URL identifying the publication in the public portal 


\title{
In vivo Motion Correction in Super Resolution Imaging of Rat Kidneys
}

\author{
Iman Taghavi ${ }^{1}$, Sofie Bech Andersen ${ }^{2,4}$, Carlos Armando Villagómez Hoyos ${ }^{3}$, \\ Michael Bachmann Nielsen ${ }^{2,4}$, Charlotte Mehlin Sørensen ${ }^{2}$ and Jørgen Arendt Jensen ${ }^{1}$ \\ ${ }^{1}$ Center for Fast Ultrasound Imaging, Department of Health Technology, \\ Technical University of Denmark, DK-2800 Lyngby, Denmark \\ ${ }^{2}$ Department of Biomedical Sciences, University of Copenhagen, DK-2100 Copenhagen, Denmark, \\ ${ }^{3}$ BK Medical, DK-2730 Herlev, Denmark, \\ ${ }^{4}$ Department of Diagnostic Radiology, Copenhagen University Hospital, DK-2100 Copenhagen, Denmark
}

\begin{abstract}
Super Resolution (SR) imaging has the potential of visualizing the micro-vasculature down to the $10 \mu \mathrm{m}$ level, but motion induced by breathing, heartbeats, and muscle contractions are often significantly above this level. The paper therefore introduces a method for estimating tissue motion and compensating for this. The processing pipeline is described and validated using Field II simulations of an artificial kidney. In vivo measurements were conducted using a modified bk5000 research scanner (BK Medical, Herlev, Denmark) with a BK 9009 linear array probe employing a pulse amplitude modulation scheme. The left kidney of ten Sprague-Dawley rats were scanned during open laparotomy. A 1:10 diluted SonoVue contrast agent (Bracco, Milan, Italy) was injected through a jugular vein catheter at $100 \mu \mathrm{l} / \mathrm{min}$. Motion was estimated using speckle tracking and decomposed into contributions from the heartbeats, breathing and residual motion. The estimated peak motions and their precisions were: Heart: Axial: $\mathbf{7 . 0} \pm \mathbf{0 . 5 5} \mu \mathrm{m}$, Lateral: $38 \pm \mathbf{2 . 5}$ $\mu \mathrm{m}$, Breathing Axial: $5 \pm \mathbf{0 . 2 9} \mu \mathrm{m}$, Lateral: $\mathbf{2 6} \pm \mathbf{1 . 3} \mu \mathrm{m}$, and Residual: Axial: $30 \mu \mathrm{m}$, Lateral: $90 \mu \mathrm{m}$. The motion corrected micro-bubble tracks yielded SR images of both bubble density and blood vector velocity. The estimation was, thus, sufficiently precise to correct shifts down to the $10 \mu \mathrm{m}$ capillary level. Similar results were found in the other kidney measurements with a restoration of resolution for the small vessels demonstrating that motion correction in 2-D can enhance SR imaging quality.
\end{abstract}

\section{INTRODUCTION}

Ultrasound Super Resolution Imaging (SRI) has been introduced over the last six years by a number of research groups [1-7]. The method uses tracking of micro-bubble (MB) contrast agents to visualize the micro-vasculature down to vessel sizes of 2-20 $\mu \mathrm{m}$, and should under ideal circumstances be capable of depicting capillary networks. The images are acquired over several minutes, over which time the object is considered stationary. This is only possible for fixated objects like a rat brain [5] or a fixated mouse ear [4]. Motion is induced in the tissue from breathing, the beating heart, and from muscle contractions [8], and it can be several times larger than the resolution attainable in super resolution imaging. The tissue motion is in all directions and also deforms the tissue, so it is both spatially and temporally variant. It is, thus, important to estimate the motion field and accurately time align it at the precise spatial locations.
Lately, a number of groups have also addressed motion correction to relax the requirement on tissue stationarity. Hansen et al [9] showed that motion correction of data from a rat kidney could increase precision from 22 to $8 \mu \mathrm{m}$ for a single vessel with the motion estimated at a single location and for a single vessel example. In [10] motion effects were reduced by excluding frames with a too high motion from breathing yielding a resolution of $2.1 \mu \mathrm{m}$ in the axial direction and $6.1 \mu \mathrm{m}$ in the lateral direction. Rigid motion was assumed throughout the image.

In [11] a rigid motion correction for the whole image was employed based on a phase correlation method, which visually gave sharper images and more narrow vessels. Two stage motion correction was applied in [8] with a combination of affine registration for the global motion, and non-rigid registration for estimating the local deformation of tissue. This reduced the width of the micro-vessels by a factor of roughly 1.5. The approach was also simulated for three-dimensional motion correction in [12]. Piepenbrock et al. [13] used a flow approach to find the velocity field and suppress the influence from the bubble response on the motion estimation. The approach was tried on a single mouse tumor.

Recently, [14] investigated a more advanced imaging technique by combining ultrasound microscopy and dualfrequency imaging technique for obtaining a high signal-tonoise ratio (SNR) and a high frame rate. Segments of 100 contrast images with a frame rate of $500 \mathrm{~Hz}$ were acquired, followed by B-mode images with a frame rate of $5 \mathrm{~Hz}$. The B-mode frame rate was only sufficient to capture the motion from breathing and more rapid movements had to be discarded. None of the previous studies have addressed the importance of the spatial and temporal difference between the motion estimates in B-mode images and the contrast data, when the two images are acquired at two different time points.

The approach taken in this paper is to estimate the full motion for all frames without restrictions on the motion, and then co-register the estimated tracks to a reference frame as described in Section II. The paper is an expanded version of the conference paper [15] with an optimized processing, motion estimation as a function of both space and time, more comprehensive statistics, and results from ten rats as described 
in Section III. The major contribution is to demonstrate the importance of the spatial and temporal variation, how it can be properly found through interpolation, and give the precision of the motion components in vivo. The kidneys of Sprague-Dawley rats were selected as scan objects, as they both experience motion from breathing, the heartbeat, and limited muscular motion making them suitable for controlled, repeatable, and realistic experiments. The kidney is also a highly perfused organ, which roughly receives $20 \%$ of the volume flow for the rat with a vascular structure including both large and small vessels, making it ideal for investigating the possibility of identifying the whole vascular tree at all levels.

The SR pipeline is validated on simulated data and on data from the left kidney of Sprague-Dawley rats as explained in Section IV. The final results demonstrate that resolution can be maintained, although the motion is 5 to 10 times larger than the precision attained. Finally the method is discussed in Section V.

\section{Methods}

This section introduces the various methods in the motion correction SRI pipeline shown in Fig. 1. The non-linear imaging scheme is introduced in II-A, and the basic processing pipeline is presented. The method for determining motion and correcting it is explained in Section II-C. Finally, the image formation is presented in Section II-D.

\section{A. Data acquisition and imaging scheme}

The data was acquired using a modified bk5000 scanner (BK Medical, Herlev, Denmark) equipped with a research interface, developed specifically for this project, for the live streaming of the data to a disk. This allowed long acquisitions for 10 minutes of beamformed radio frequency (RF) data. A "hockey stick" X18L5s transducer (BK 9009, BK Medical, Herlev, Denmark) was employed for imaging. This linear array probe with 150 elements covers an imaging width of $24 \mathrm{~mm}$, which was sufficient for scanning a full rat kidney. The height of the aperture was $3.4 \mathrm{~mm}$, and the elevation focus was at $20 \mathrm{~mm}$ for an elevation F-number (depth divided by active aperture width) of 5.9 .

A pulse amplitude modulation sequence was used for imaging [16] with a transmit frequency of $6 \mathrm{MHz}$. It employed a sliding aperture approach of 25 elements with a focal depth of $10 \mathrm{~mm}$. Each active aperture emitted three times, one with full amplitude and two with half amplitude. This combination of emissions gave the amplitude-modulated response for enhancing the non-linear signal from the contrast agent. The active aperture then slided across the array for a total of 91 active sub-apertures. This is visualized in Fig. 2, and is referred to as the contrast sequence. After this, a B-mode image was acquired. It employed the same 91 active subapertures, however emitted only once per active sub-aperture. This resulted in $3 \cdot 91+91=364$ emissions per full sequence. The transmit voltage was kept low with a corresponding mechanical index (MI) of 0.2 to avoid disrupting the MBs.

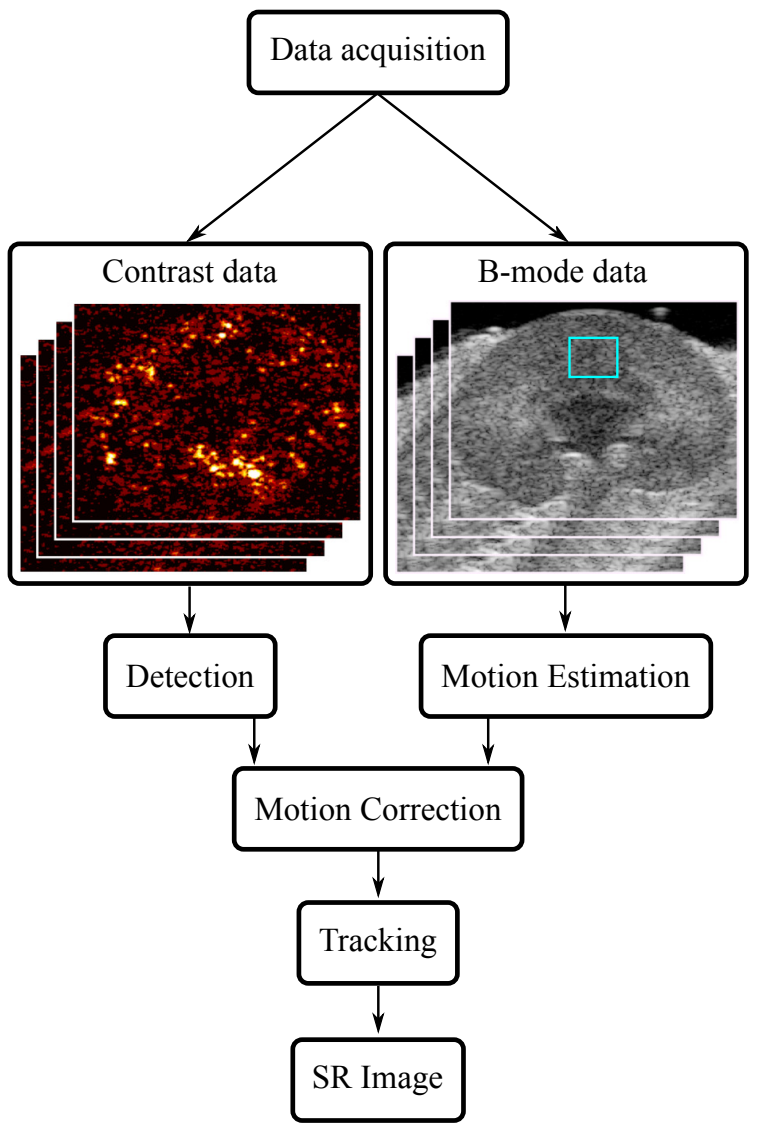

Fig. 1. Block diagram of the processing scheme used in the paper. The blue rectangle in the B-mode image indicates a local region used to estimate the dynamics of the motion.

The pulse repetition frequency $f_{p r f}$ was $19.6 \mathrm{kHz}$ for a frame rate of $53.85 \mathrm{~Hz}$.

The MB dilution of 1:10 was chosen for the individual MBs' point spread function to be distinguishable in each image frame. Due to the contrast dilution, an acquisition time of 10 min was chosen, as pre-trials showed this scan duration was needed to sufficiently fill the dense vasculature of the kidney with MBs [17].

Three emissions of the contrast sequence corresponded to the same image line as one emission in the B-mode sequence. The time difference of formation of each line in the contrast image and its next B-mode image is easily measurable from Fig. 2. The time difference between the $n^{\text {th }}$ line of a contrast image and the $m^{\text {th }}$ line of its next B-mode image is:

$$
t_{d}(n, m)=\frac{3 N_{\text {lines }}-3 n+m+1}{f_{\text {prf }}},
$$

where $n, m \in\left\{1,2, \ldots, N_{\text {lines }}\right\}$ and $N_{\text {lines }}$ is the number of lines in the image. This time difference plays an important role in applying the estimated motion to the correct positions in the contrast image.

An example of B-mode and contrast images are shown in Fig. 1. The B-mode image has a poor visualization of the contrast agent, but clearly shows how the anatomical structures move during the scans. The contrast image clearly shows the different MBs with a high contrast. Thus, the combination of 


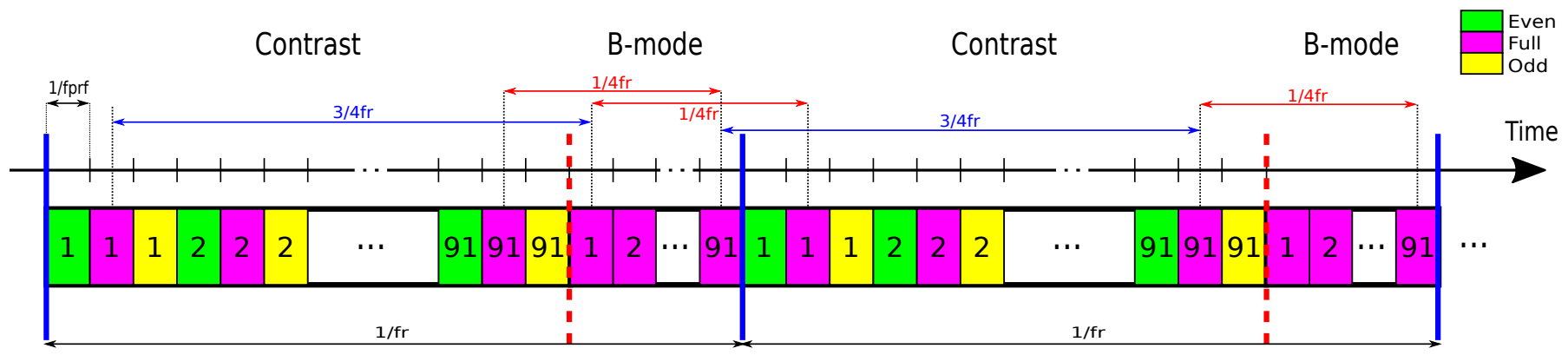

Fig. 2. Imaging scheme used in the in vivo studies. Each contrast sequence consisted of $3 \times 91$ emissions followed by a B-mode sequence consisting of 91 emissions. While each image line, in the contrast and B-mode images refers to the same lateral position, there was a unique delay between the time of formation of each line in the contrast images to the corresponding line of the B-mode image varying from $1 /\left(4 f_{r}\right)$ to $3 /\left(4 f_{r}\right)$.

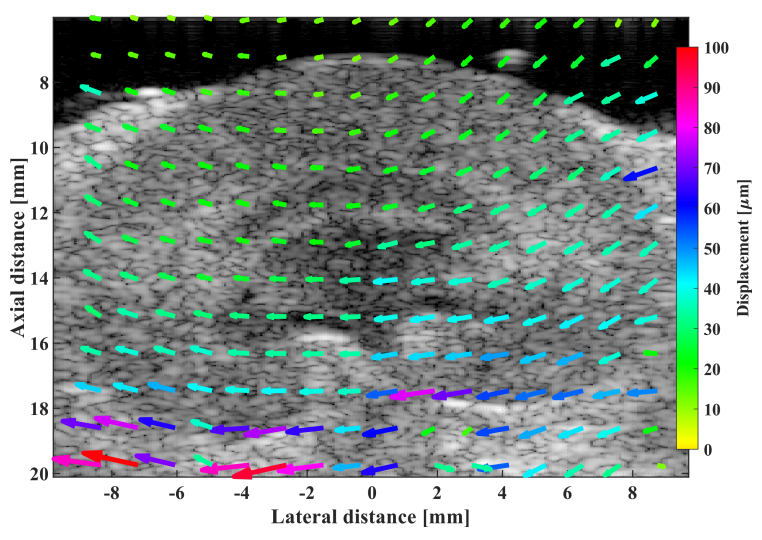

Fig. 3. Estimated motion field for a single frame. The arrows indicate direction and magnitude of the motion and were found from the interpolated motion field.

the two images yields both anatomy motion and contrast agent location.

\section{B. Detection}

Detection is required to extract the centroid of the MB's positions from the contrast images. This process consists of two parts: 1) "SNR enhancement" by e.g. simple thresholding, spatial filtering (e.g. Gaussian, Laplacian of Gaussian), spatiotemporal filtering (e.g. singular value decomposition) [18, 19], or model fitting [20] and 2) "localization" using peak detection, centroid estimation methods (.e.g. weighted centroid) [20, 21], or learning-based methods [22, 23]. A single or a combination of these methods can be employed for detection.

The contrast images only had a few quantization levels yielding a low SNR. The detection threshold was experimentally adjusted to reduce background noise, and the threshold was 1.1 for these data sets, which had only positive integer values up to 5. To improve SNR, a Gaussian filter was applied to the contrast image after thresholding. The kernel size of the symmetric Gaussian filter was 7 pixels with a standard deviation of 1 pixel, where pixel size in the axial direction was $24 \mu \mathrm{m}$ and $80 \mu \mathrm{m}$ in the lateral direction. Then, the centroids of the MB positions were estimated using the weightedcentroid algorithm.

\section{Motion estimation and correction}

The tissue motion was estimated from the B-mode image using speckle tracking [24] on the envelope data. Regions in the images were correlated to corresponding regions in a selected reference frame number, and motions in the axial and lateral directions were found. Speckle tracking using the RF data was also attempted, but the lateral sampling density was too low to yield acceptable lateral estimates.

The entire kidney was in the field-of-view, and the motion was not uniform across the whole organ as seen in Fig. 3. Hence, two different spatial regions do not have the same displacement, and a single local motion estimation cannot be applied across the whole kidney. It was therefore divided into smaller spatial blocks for block-wise processing. The Bmode image was divided into $80 \%$ overlapped $3 \times 3 \mathrm{~mm}^{2}$ local regions, and the estimated motion signal was assigned to the center of each region $\vec{r}_{k}=\left(z_{k}, x_{k}\right)$. The collection of all motion signals in the form of $\vec{F}\left(t_{k}, \vec{r}_{k}\right)$ provided a discrete motion field through the entire image. Fig. 3 illustrates a snapshot of the motion field with arrows on top of the corresponding B-mode frame. Notice how the estimated displacements vary spatially, as opposed to a rigid structure.

The motion field was calculated with the following assumptions:

- The local regions were assumed small enough, so that the motion was spatially invariant inside the regions. This assumption depends on the type of material and application. Although a kidney is not a rigid material, the motion through a small local tissue region has the same magnitude and direction.

- The frame rate was high enough to capture the fastest variation of the motion in the temporal domain. The acceleration of the motion depends on the source of the motion, and the fastest source for the motion was the heart. According to the Nyquist theorem, the frame rate should be at least two times the maximum frequency in the heartbeat spectrum to capture the fastest transitions in the motion. This was investigated in Section IV-B.

The motion estimates were applied on the MB locations found by the processing pipeline. The time point for the motion depends on the location in the image, as a linear sweep of the beams were conducted from the most positive lateral locations to the most negative location as described in Section II-A. The motion estimation only gave one estimate per frame, and the motion time series was spline interpolated by a factor of 20 to yield a higher time resolution. A linear interpolation between these time points was then made to match the exact 
time location corresponding to the spatial location of the MB. Motion from one frame to the next easily change with 100$200 \mu \mathrm{m}$, and this time interpolation was therefore essential to attain a co-registration better than $10 \mu \mathrm{m}$.

Finally a 2-D spline interpolation was performed on the time interpolated motion field to yield the exact motion for the spatial position of the detected MB, and this was used in the motion correction to align the positions of the MB to the reference frame. Motion estimates for one of the regions in the medulla was used to study the dynamics and precision of the motion estimation algorithm as described in Section IV-B.

Three different scenarios were investigated for image motion correction: 1) No motion correction where the images were formed without compensating for motion. 2) Local motion correction where a single region was selected for motion estimation. The estimated motion for this region was interpolated in time to account for the different motions at different times. The exact acquisition time for the MB was found, and the estimated motion for this time was used for compensation. 3) Global motion correction entails estimation of the motion in all image regions, time interpolation, and use the motion found dependent on the MB image position and thus time for acquisition.

\section{Image formation}

The next step was to generate MB tracks. The bubble position was adjusted by the motion estimated in Section II-C, and the motion corrected bubbles in the next frame were found to determine the bubble closest to the current bubble. The search for the next bubble was restricted to the region given by $v_{s} / f_{r}$, where $v_{s}$ was the maximum allowed MB velocity and $f_{r}$ was the frame rate of the sequence. The maximum velocity was selected to $v_{s}=13.5 \mathrm{~mm} / \mathrm{s}$. The selected bubble was then added to the track and removed from the list of bubbles in the frame. The search was continued until no bubble satisfying the criteria was found. Tracks with less than three bubbles were considered noise outliers and were not stored.

Images were then formed from these tracks by inserting the bubble tracks into a high resolution image with a pixel size of $5 \mu \mathrm{m}$. Each track was inserted into the images by drawing an anti-aliased vector between the different positions in the track using the algorithm developed in [25]. The vector was added to the current content of the image, and this finally yielded a bubble density image. The velocity of the bubbles was found by taking the time derivative of track locations, which yielded both the axial and lateral velocities. The velocity tracks were inserted into an axial and a lateral velocity image by drawing anti-aliased velocity weighted lines at the track positions. A weighting image was also drawn, and the mean velocity at each pixel in the velocity images were found by dividing with the weighting image, if it was different from zero. The axial and lateral velocity images were then combined to yield a Vector Flow Image (VFI) of the bubble velocity. The resulting images are presented in Section IV-C.

\section{E. Resolution estimation}

The Fourier ring correlation (FRC) criterion was used to measure the resolution $[26,27]$. Using the threshold criteria to

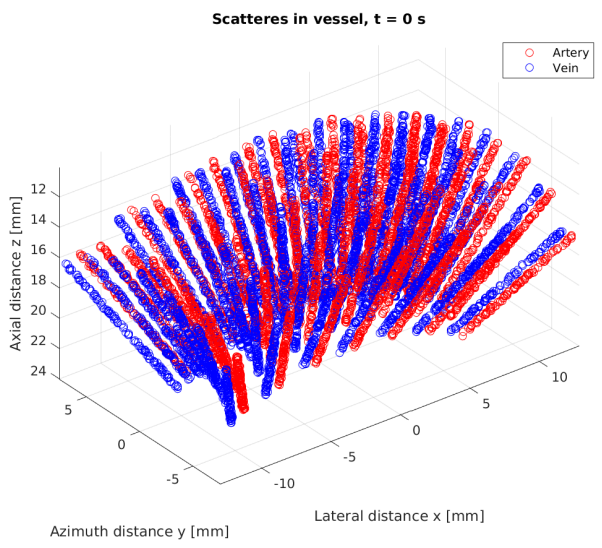

Fig. 4. Geometry of the in silico rat kidney phantom. The red structures have flow up-wards and the blue down-wards.

define resolution is controversial [28], and this study therefore uses a bit-based information threshold curves for the FRC threshold level as discussed in [28]. The SR images are split into two images by inserting every other track into an image. The split images were normalized to their maximum intensity values, and their intensity equalized using the imhistmatch.m function in Matlab. The FRC curve of two images was calculated based on [26] without zero-padding, and the resolution was estimated as the inverse spatial frequency, where the onebit threshold curve crossed the FRC curve [28].

\section{EXPERIMENTAL DATA}

The motion correction method was investigated using both simulated data as described in Section III-A as well as in vivo data from Sprague-Dawley rats as described in Section III-B.

\section{A. Simulation phantoms}

The linear part of the pulse amplitude sequence has been simulated using Field IIpro [29-31] to generate reference data, where the positions of the scatterers are known. The first phantom contains a matrix of point targets located at a distance of $2 \mathrm{~mm}$ in both the axial and lateral direction.

The second phantom was based on the dimensions of a rat kidney and is shown in Fig. 4. It mimics the flow in small arteries and veins, where the arterial flow was towards the perimeter and the return venous flow was in the opposite direction. A sparse distribution of scatterers were located randomly in the individual tubes with parabolic velocity profiles and a Gaussian distributed scattering amplitude. The scatterers moved along the tube axis and were returned in the adjacent tube, when they reached the tube's end. The various dimensions of the phantom are listed in Table I. A slice of the tubes in the elevation direction of the probe was selected in this simulation. Stationary scatterers surrounding the tubes were also simulated to mimic the kidney tissue. The evolution of scatterer position was given by

$$
\vec{r}\left(i, t+T_{p r f}\right)=\vec{r}(i, t)+T_{p r f} \vec{v}_{f}(\vec{r}(i, t), t)+\Delta \vec{r}_{m}(t),
$$

where $\vec{r}(i, t)$ is the position of scatterer $i$ at time $t, T_{p r f}$ is the time between pulse emissions, $\vec{v}_{f}(\vec{r}(i, t), t)$ is the velocity of 
TABLE I

PARAMETERS USED IN THE IN-SILICON RAT KIDNEY PHANTOM.

\begin{tabular}{|l|c|}
\hline Parameters & Value \\
\hline Tube length & $10 \mathrm{~mm}$ \\
Tube radius & $100 \mu \mathrm{m}$ \\
Velocity & $2 \mathrm{~mm} / \mathrm{s}$ \\
Number of scatterers & 20 per tube \\
Number of tube pairs & 49 \\
Angle between tube pairs & $10^{\circ}$ \\
Distance between pairs & $1 \mathrm{~mm}$ \\
\hline
\end{tabular}

the scatterer due to the blood flow, and $\Delta \vec{r}_{m}(t)$ is the motion of the whole kidney. For the stationary scatterers surrounding the vessel $\vec{v}_{f}(\vec{r}(i, t), t)=0$.

A realistic motion $\Delta \vec{r}_{m}(t)$ of the whole phantom was introduced from a motion estimation on the in vivo measurement. This ensured that both stationary tissue and tube locations varied across the heart and breathing cycles for validation of the motion estimation and motion correction algorithms. Only a linear simulation of the returned signal was conducted, and only the first harmonic was used in processing these data.

\section{B. Animal preparation and experiment setup}

The motion study was performed on 10 healthy male Sprague-Dawley rats according to protocols approved by the Danish National Animal Experiments Inspectorate. The experiments were conducted at the University of Copenhagen. The ethical standards of the university complies with those of the National Institutes of Health and all procedures were performed accordingly. The animals were housed at the animal facility at the Department of Experimental Medicine. They were held in a 12/12-hour light/dark cycle and could freely access drinking water and a standard chow. Appropriately trained caretakers were responsible for the animal welfare until use.

Induction of anesthesia was done with 5 percent isoflurane. After tracheotomy, the animals were connected to a ventilator (Ugo Basile, Gemonio, Italy) with a respiration cycle of 72 respirations/minute. An adequate level of anesthesia was maintained with 1-2 percent isoflurane, and the muscle relaxant Nimbex (cisatracurium, $0.85 \mathrm{mg} / \mathrm{ml}$, GlaxoSmithKline, London, United Kingdom) was continuously administered at $20 \mu \mathrm{l} / \mathrm{min}$. Jugular vein catheterization was done with polyethylene catheters (PE-10), which were then used for infusion of MBs, isotonic saline, and Nimbex. A polyethylene catheter (PE-50) in the left carotid artery and a Statham P23$\mathrm{dB}$ pressure transducer (Gould, Oxnard, CA, USA) was used to monitor the arterial blood pressure. With the animals in the supine position on a heating pad, the left kidney was exposed through open surgery. A metal retractor held the diaphragm to expose the kidney further and reduce respiratory motion. The transducer was placed on the lateral kidney surface and held by a fixated stage. Gel was used for interface coupling. The diluted (1:10) MBs (Sonovue, Bracco Imaging, Milan, Italy) were injected at $100 \mu \mathrm{l} / \mathrm{min}$., and the 10-minute data recording started when the MBs started appearing on the scanner display. Due to the floating of MBs in the syringe, a custom-built device turned the syringe 180 degrees every 60 seconds.
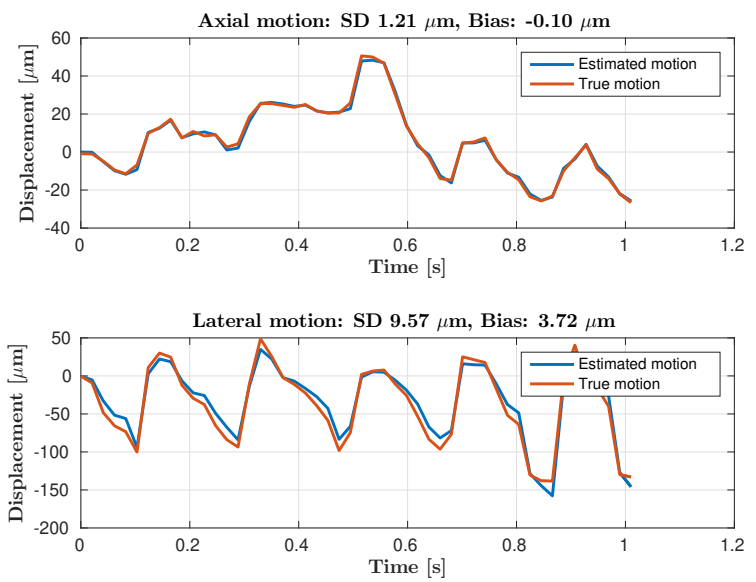

Fig. 5. Estimation of the motion from the B-mode image in the axial (top) and lateral direction (bottom), where the red curve is the true motion and the blue is the estimated motion.

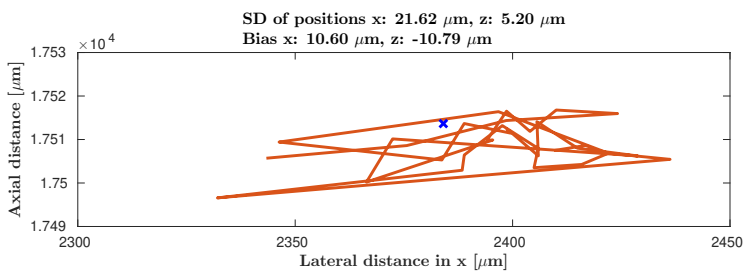

Fig. 6. Localization of a scatterer after motion compensation. The blue cross indicates the true position and the red lines indicate the motion corrected positions.

Each rat was scanned three times. First, a baseline scan was conducted. Thereafter, five rats had the left renal artery clamped, and five rats had the left renal vein clamped for 45 minutes. A second scan was performed at the onset of reperfusion after the clamp was released. The last scan was performed in a steady state, one hour after clamp release. The rats were euthanized after the scans. The data has also been used in [32] to characterize the effects of acute renal ischemia on the renal vasculature using SR imaging.

\section{RESULTS}

\section{A. Simulation validation}

The basic localization precision of stationary targets was investigated using the matrix phantom. The axial bias is 0.48 $\mu \mathrm{m}$ and the lateral is $-1.46 \mu \mathrm{m}$. The corresponding standard deviations (SD) are $10.7 \mu \mathrm{m}$ and $20.5 \mu \mathrm{m}$.

The motion of the in silico rat phantom has been estimated for the B-mode data and is shown for the first 50 frames in Fig. 5 in the axial (top) and lateral direction (bottom), where the red curve is the true motion and the blue is the estimated motion. The axial SD is $1.21 \mu \mathrm{m}$ and the lateral is slightly higher at $9.57 \mu \mathrm{m}$.

These motions estimates have been used to correct the position of a non-flowing scatterer shown in Fig. 6, where the blue cross shows the position of the scatterer in the first reference frame. The location in the subsequent frames have been corrected by the estimated motion and this yields the tracks indicated by the red lines. The precision or SD of the target location after correction is then $5.2 \mu \mathrm{m}$ in the axial and 

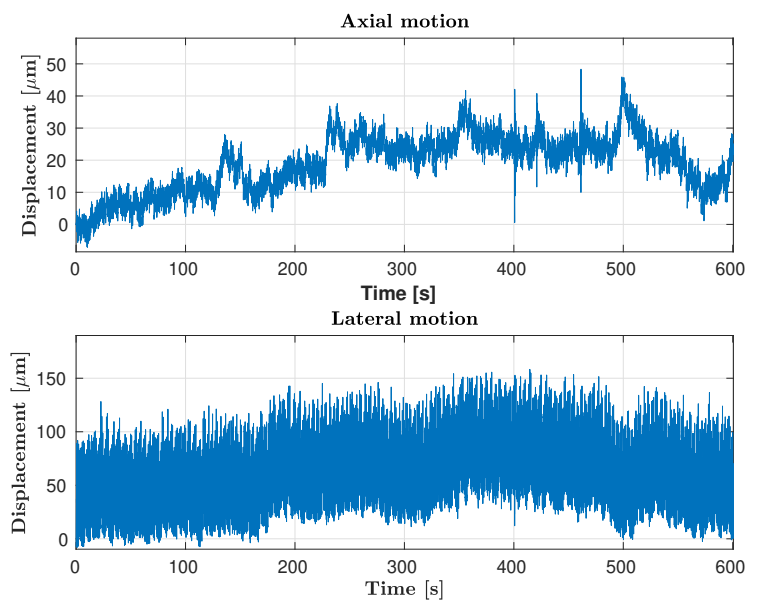

Fig. 7. Motion in the axial (top) and lateral direction (bottom) throughout the full experiment.
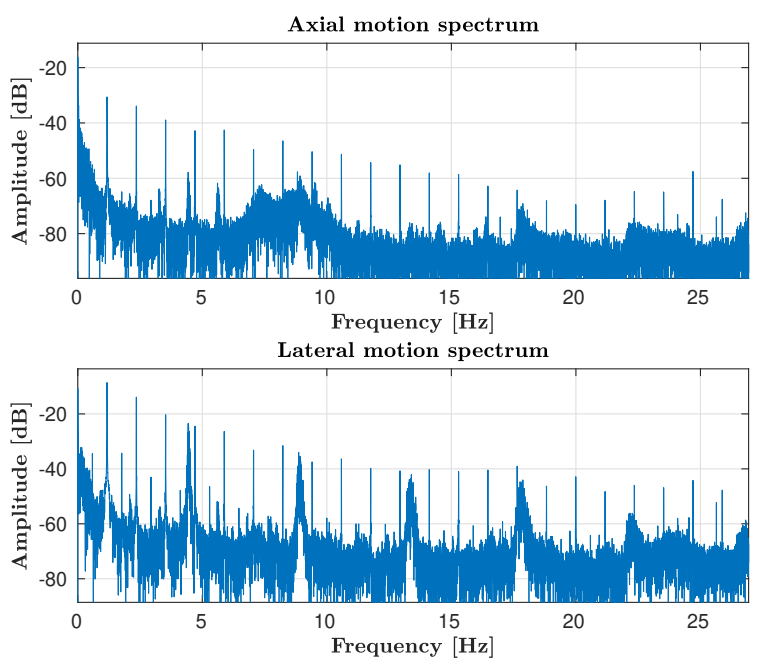

Fig. 8. Spectral decomposition of the motion signal in the axial (top) and lateral direction (bottom).

$21.6 \mu \mathrm{m}$ in the lateral direction with biases of $-10.8 \mu \mathrm{m}$ and $10.6 \mu \mathrm{m}$.

\section{B. Precision of in vivo motion estimation}

A single region in the top part of the kidney in the medulla was selected for investigating the precision of the motion estimation. The motion in both the axial and lateral directions for all ten minutes of the experiment for a single rat is shown in Fig. 7. This is a combination of motion from the beating heart, the forced ventilation (breathing), smooth muscle contractions, and over all movement, which cannot readily be separated out. A Fourier decomposition gives the spectra shown in Fig. 8 with amplitudes in $\mathrm{dB}$, where the axial spectrum is shown on the top and the lateral on the bottom. Three distinct Fourier series can be seen in both of them. First, a large component around $0 \mathrm{~Hz}$ stems from the overall drift in the data. The second series is from the breathing motion. This is controlled mechanically at a fixed rate, and the harmonics of this motion has a very narrow bandwidth around harmonics of the 70.5 Beats Per Minute (BPM), and more than 20 harmonics can be
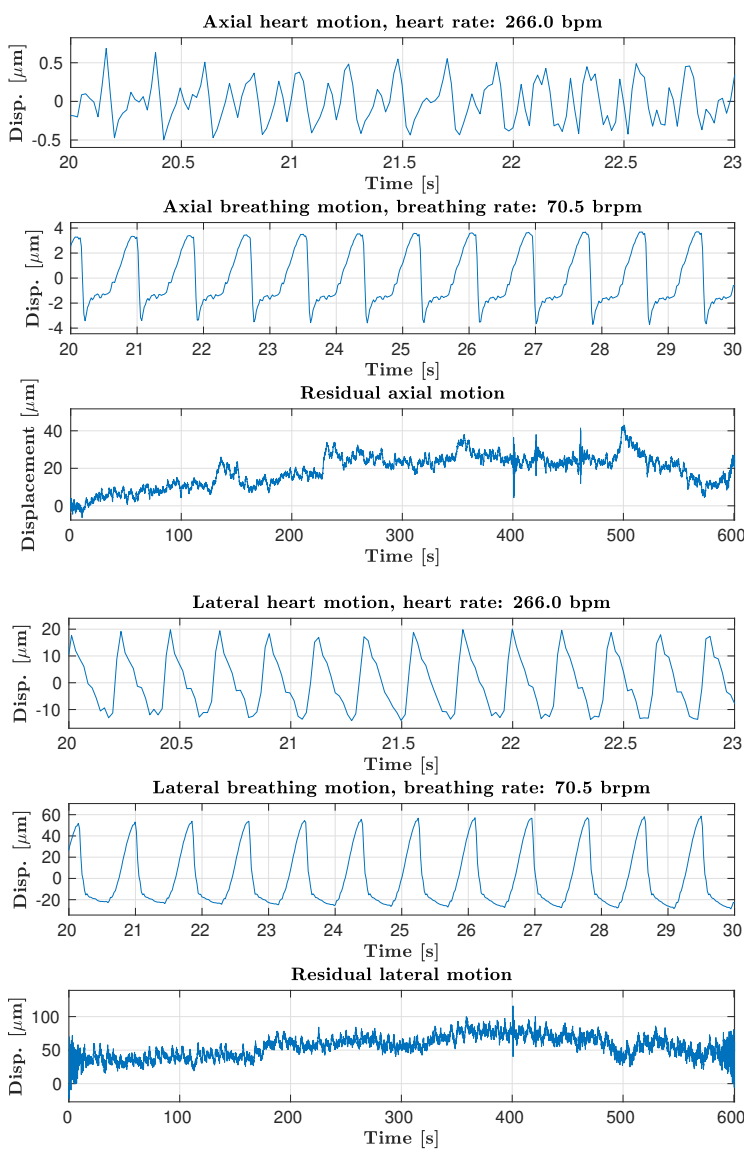

Fig. 9. Separation of the three motion components for the axial motion (top) and the three components for the lateral motion (bottom)

seen in both spectra. The last series is from the heart motion, which has a lower amplitude and the components are placed around harmonics of the heart rate of approximately $353 \mathrm{BPM}$, where 5 harmonics can be seen in the lateral motion signal. The harmonics are spread over a larger bandwidth, due to the physiological variation of the heart rate.

These three Fourier series can be separated to yield the three independent motion signals as shown in Fig. 9, where the top three graphs display the components for the axial motion. The top graph is when the heartbeat components have been kept by isolating the first four harmonics around the heart rate. The middle graph is when isolating harmonics for the breathing for the first 18 harmonics. The lowest graph is for the residual signal, where the two other Fourier series have been subtracted.

The repetitiveness of the heartbeat and the breathing can be used for aligning all the responses and yield a mean response and its precision. This is shown in Fig. 10 for the heartbeat motion and in Fig. 11 for the breathing. In this example the heart motion is around $\pm 1 \mu \mathrm{m}$ (axial) and -15 to $+20 \mu \mathrm{m}$ (lateral), and the precisions are 0.348 and $1.89 \mu \mathrm{m}$ including the physiological variation of the heartbeat. The breathing motion spans from $-20 \mu \mathrm{m}$ to $+60 \mu \mathrm{m}$ with precisions of 0.271 and $1.64 \mu \mathrm{m}$. The residual motion shown in Fig. 9 is larger with deviations up to nearly $100 \mu \mathrm{m}$. For vessels to align up and maintain super resolution it is, thus, very important to 

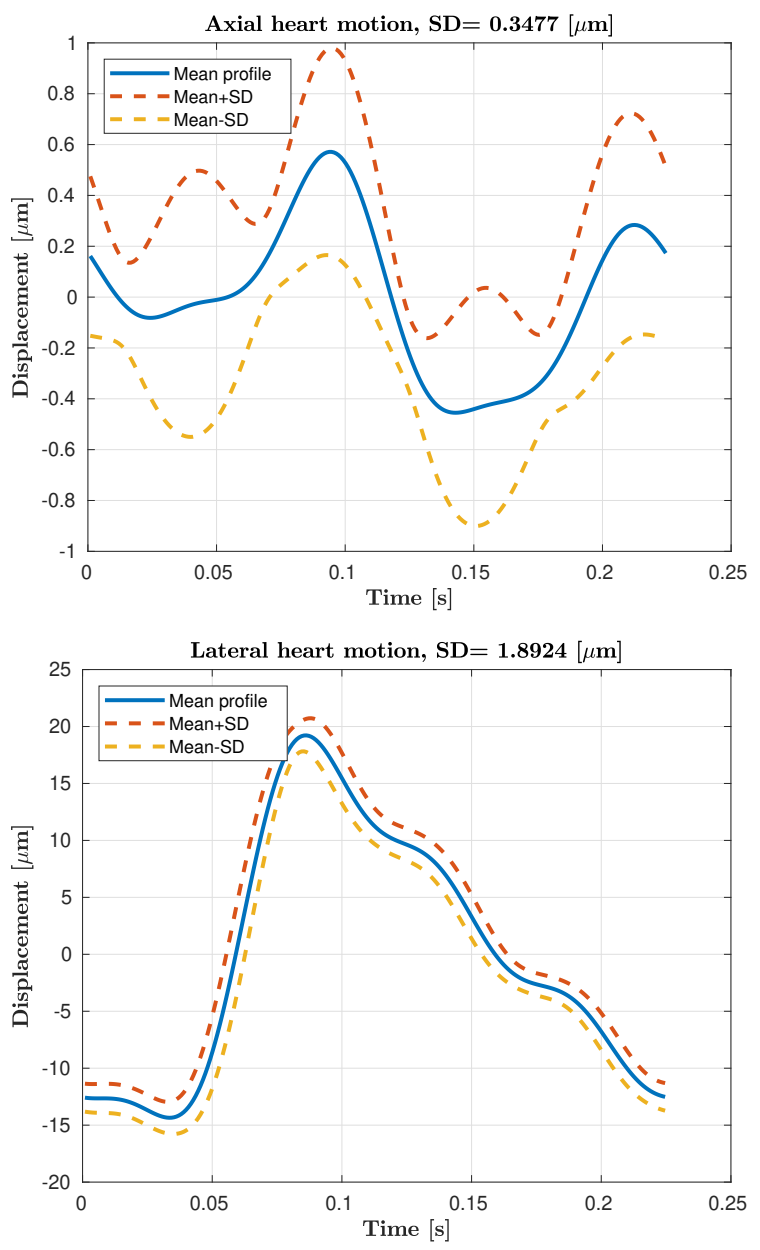

Fig. 10. Mean heartbeat motion for the axial (top) and lateral (bottom) direction. The blue solid line denotes the mean value across all cycles and the dashed lines show one SD.

TABLE II

PRECISION ESTIMATES COMBINED FOR ALL RAT EXPERIMENTS. ALL VALUES IN $\mu \mathrm{M}$.

\begin{tabular}{|l|c|c|c|}
\hline Motion type & Minimum & Maximum & Mean \\
\hline Heartbeat, axial & 0.069 & 7.858 & 1.992 \\
\hline Breathing, axial & 0.062 & 4.630 & 1.455 \\
\hline Heartbeat, lateral & 0.431 & 19.923 & 6.562 \\
\hline Breathing, lateral & 0.277 & 20.315 & 5.438 \\
\hline
\end{tabular}

compensate for the combined motion.

The experiment has been repeated for the ten different rats, with three measurements on each rat as explained in Section III-B. A similar region and reference image were selected for each measurement. All precision estimates for all ten rats and all three experiments are shown in Fig. 12 for the four different motion estimates and summarized in Table II. There is a quite wide variation in precision with estimates reaching $20 \mu \mathrm{m}$. This happens in experiments where the motion is too large for the speckle tracking to capture motion in part of the images and can be due to motion in the out-of-plane direction, or that the set-up has been touched inducing a significant kidney motion. It can, however, be seen that the precision of the motion estimation is around a factor of two lower than the $10 \mu \mathrm{m}$ size of the capillaries and often a factor of
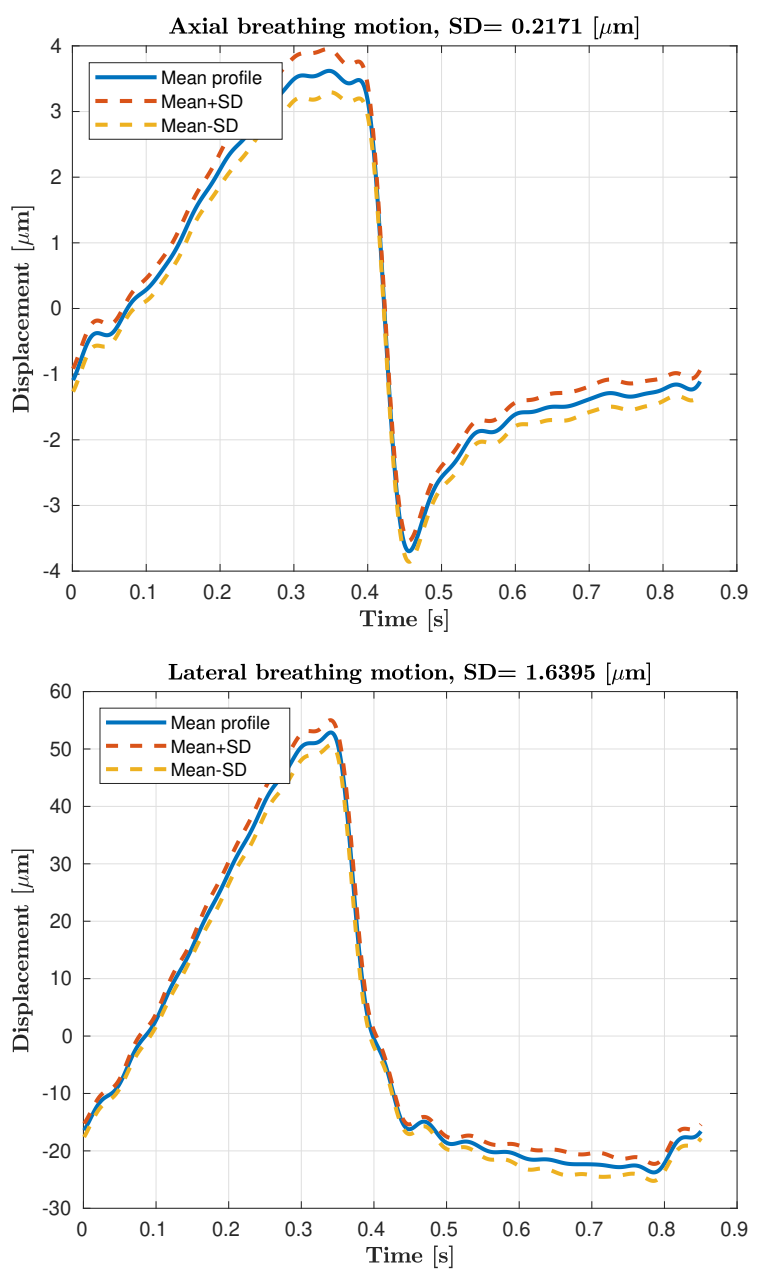

Fig. 11. Mean breathing motion for the axial (top) and lateral (bottom) direction. The blue solid line denotes the mean value across all cycles and the dashed lines show one SD.

5 lower, indicating that a motion corrected image potentially can visualize capillary flow.

\section{Motion compensated images}

The log-scaled intensity SR images without motion correction, with local motion correction, and with correction using the motion field are depicted in Fig. 13 (top). The corresponding velocity images are shown in the second row with color indicating direction and intensity magnitude, e.g. a yellow color indicates velocity from left to right in the image. The third row shows the zoomed regions $1-4$. Four markers on the intensity and velocity images were selected exactly at the same place to compare the intensity and velocity profiles of the SR images. Polynomial fitted profiles to the pixel values of the SR images are shown in the bottom row for comparing diameter and flow velocities of the selected vessels. To remove unrealistic tracks, the MBs that were not traceable for more than 5 frames as well as those that had an overall displacement less than $200 \mu \mathrm{m}$ for the whole track (stationary bubbles) were removed. Only tracks with velocities from 0 to $10 \mathrm{~mm} / \mathrm{s}$ are shown.

The effect of motion correction was investigated by looking at intensity and velocity profiles over the various cortical and 

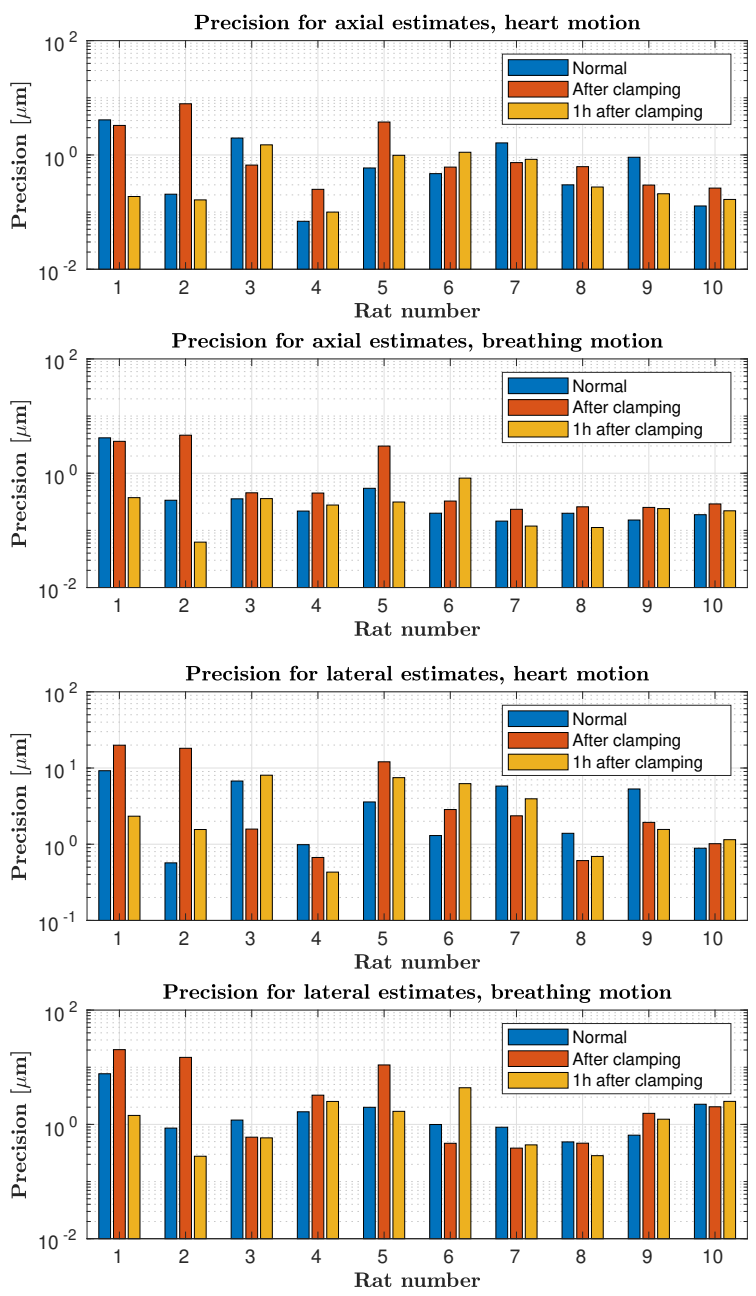

Fig. 12. Precision estimates for the various rat experiments for the axial (top) and lateral (bottom) direction.

medullary (inner part of the kidney) vessel structures shown in region 1-4 in Fig. 13. The profiles of the exact same regions on the images with and without motion correction are shown in Fig. 13 (bottom).

The FRC curves for the images in Fig. 13 with one-bit and half-bit information threshold curves are shown in Fig. 14, where the left graph is without motion correction, middle is with local motion correction, and right is with global motion correction.

Fig. 15 shows the FRC resolution with one-bit threshold for all 10 SR kidney images. It shows an improvement in the image quality after motion correction using the motion field both in the median and inter-quartile range.

\section{DISCUSSION}

In vivo measurements have been conducted on 10 partly fixated rat kidneys using an amplitude modulation non-linear imaging scheme. Estimating the motion induced from the forced ventilation and the heartbeating showed that motion in the $100-200 \mu \mathrm{m}$ range in both the axial and lateral directions were present. Such motion would limit the resolution in SR imaging to $100-200 \mu \mathrm{m}$ and the smallest vessel would not be visible. Compensating the motion in reference to one selected frame re-establishes resolution. In simulations the precision of the target location after correction is $3.9 \mu \mathrm{m}$ in the axial and $17.7 \mu \mathrm{m}$ in the lateral direction with biases of -12.4 $\mu \mathrm{m}$ and $6.5 \mu \mathrm{m}$. In general the lateral motion was estimated with a lower precision than the axial motion. This is due to employment of the signal envelope as the lateral density of lines is too low to employ cross-correlation of the RF signals. One obvious choice is to employ fast imaging like Synthetic Aperture [33] or plane wave imaging [34] for high frame rates with no restrictions on the lateral sampling density. The major drawback of this is the increase in the amount of data, as the full RF data for a number of transducer elements have to be employed. This is currently difficult with the long acquisitions used here (10 minutes), but should be implemented in future scanners. The frame rate could also be increased, if the second set of full emissions in the sequences shown in Fig. 2 could be avoided. This is, however, currently not possible, due to the processing and storage capabilities of the bk5000 scanner. The long acquisition time can potentially be optimized by using the quantitative assessment of vessel reconstruction suggested by Dencks et al. [35].

The precision of the in vivo motion was also determined by decomposing the motion signal into components from breathing, heartbeat, and residual smooth muscle motion. The breathing motion was in general larger than motion induced by the heart. The precision was $217 \mathrm{~nm}$ (axially) and 1.64 $\mu \mathrm{m}$ (laterally). The heartbeat motion had a precision of 69 $\mathrm{nm}$ (axially) and $0.98 \mu \mathrm{m}$ (laterally) including physiological variation, and it is a factor of 10 lower than the target of 10 $\mu \mathrm{m}$ for visualizing capillary flow. For all rat experiments the motion could be estimated with a precision from $69 \mathrm{~nm}$ to $20.3 \mu \mathrm{m}$ from the linear B-mode image, with a mean axial motion precision of $2.0 \mu \mathrm{m}$ in the axial direction and $6.6 \mu \mathrm{m}$ in the lateral direction.

The effect of motion correction is shown in Fig. 13. Using only a single motion correction for the whole image does not fully correct for the motion, which can be seen by comparing images in rows $b$ and $c$ for the zoomed regions. The effect of motion correction on the alignment of small vessels was especially prominent in the cortical (outer) part of the kidney in the velocity images. The results show that having a motion correction through the entire kidney will provide sharper images and more smooth intensity and velocity profiles.

Both intensity and velocity for Profile 1 shows that the diameter of the large vessel in the down-left side of the kidney was reduced from $1.3 \mathrm{~mm}$ to $0.7 \mathrm{~mm}$, and that profiles without motion correction were widely spread, resulting in a blurred image. This shows the reduction in the width of the vessel by a factor of roughly 2 . The velocity in this large vessel was around $8 \mathrm{~mm} / \mathrm{s}$. Profile 2 was placed over two vessels with a size of $500 \mu \mathrm{m}$. Profiles 3 and 4 were calculated over four smaller vessels with a diameter of less than $50 \mu \mathrm{m}$ and $250 \mu \mathrm{m}$, respectively. The intensity profiles did not show how many vessels existed in the regions. However, the velocity profiles showed the resolved small vessels. The velocity in smaller vessels of the medulla (middle part of the kidney) was measured below $1.5 \mathrm{~mm} / \mathrm{s}$, as demonstrated in Profile 3 . Profile 4 over a part of the cortical region showed a higher 

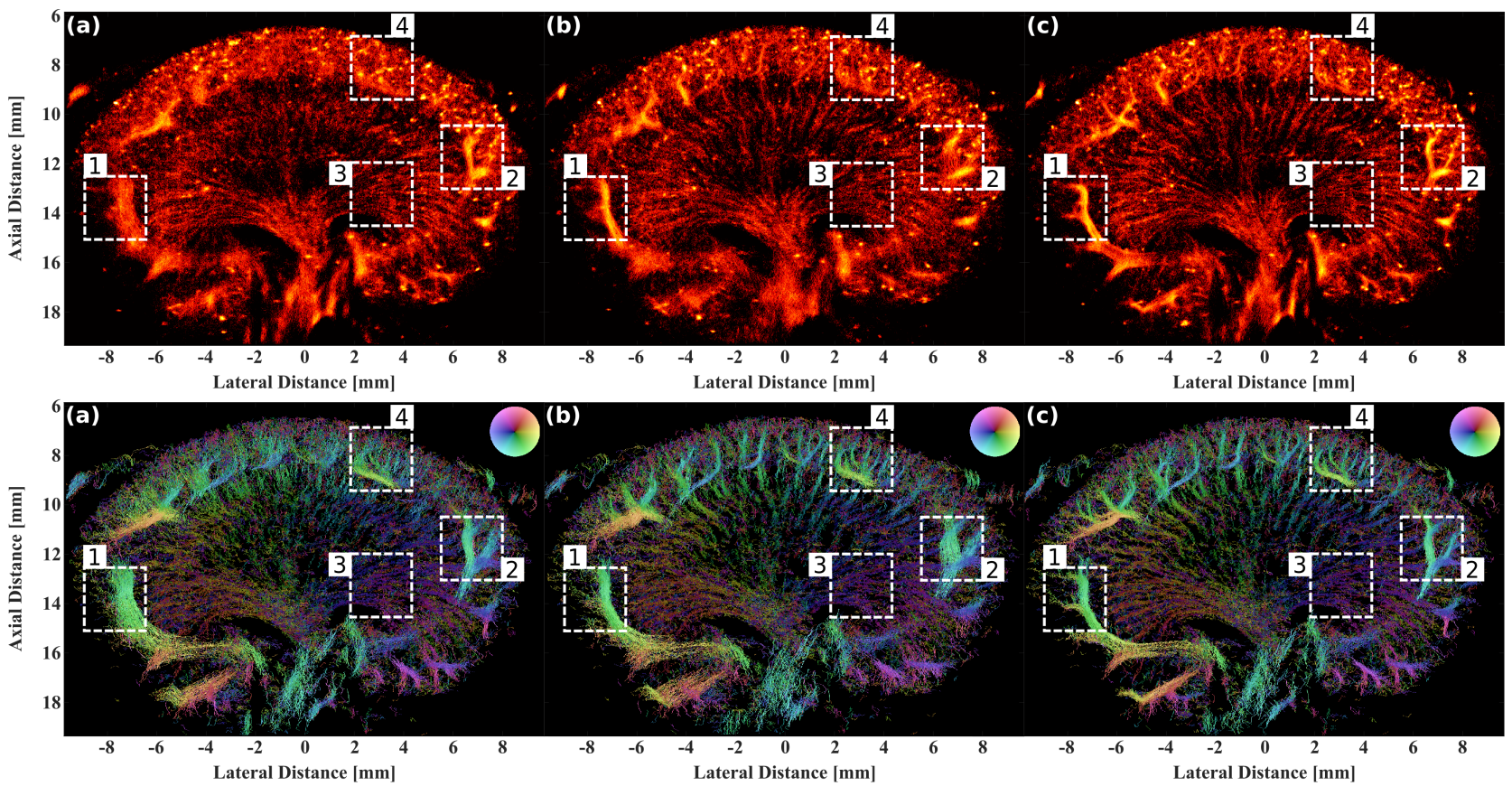

(a)
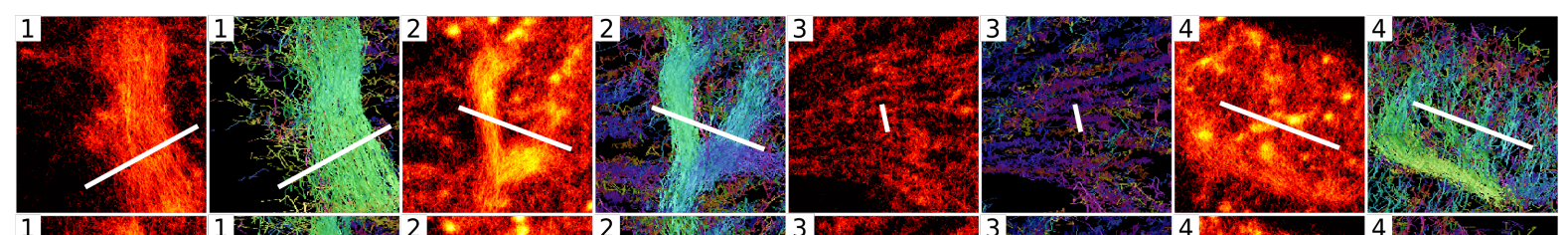

(b)
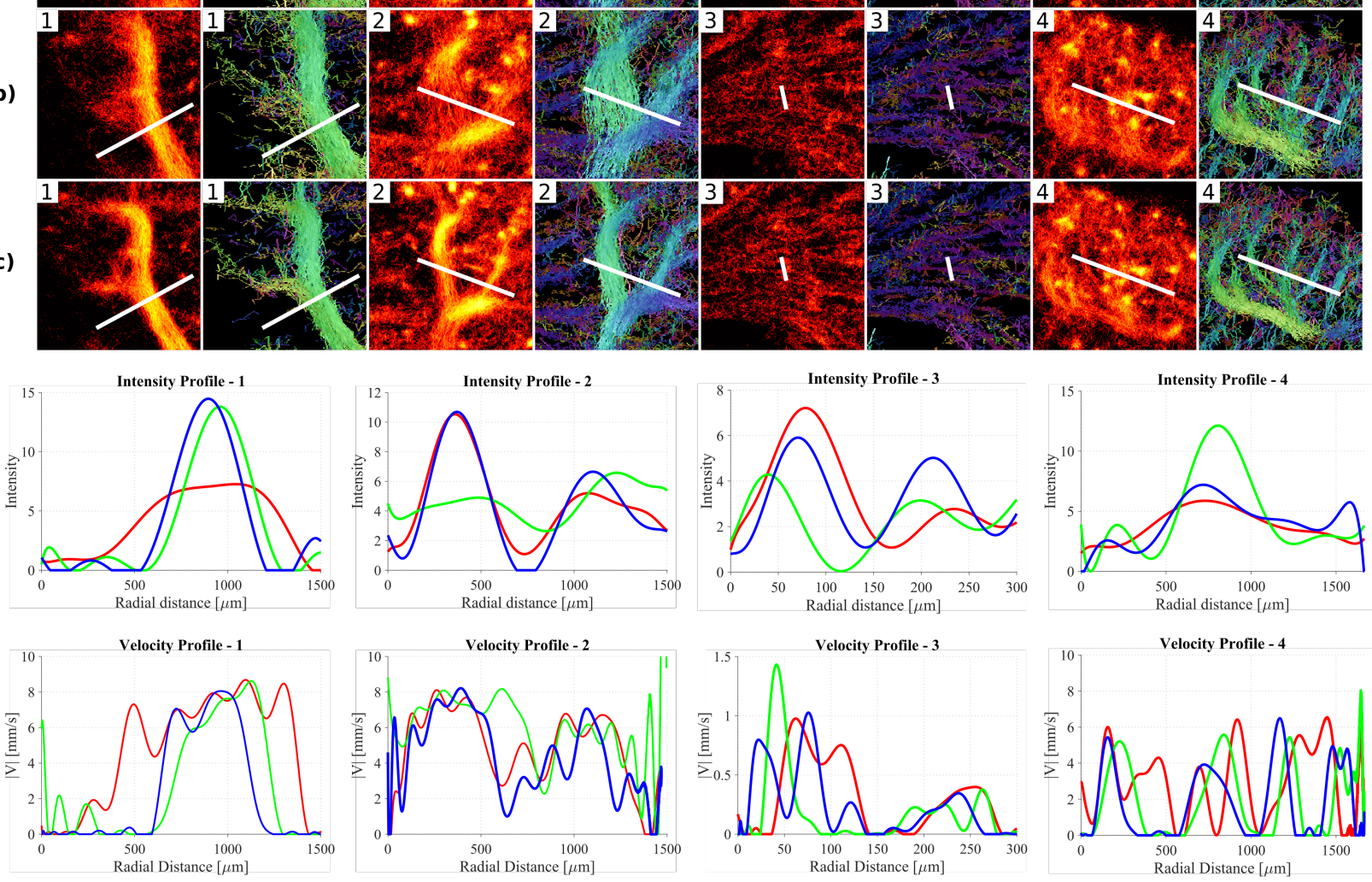

$\begin{array}{llll}\text { (a)Without motion correction } & \text { (b) Local motion correction } & \text { (c) Correction via Motion Field }\end{array}$

Fig. 13. Intensity and velocity SR images of a rat kidney (a) without motion correction, (b) with local motion correction, and (c) with correction using the motion field are shown. The intensity images show on the top row is an accumulation of all bubble positions. The second row shows the velocity images with color coded traces, where intensity is proportional to MB velocity and direction is shown as the color. Marked regions 1-4 are indicated in the top images and the third row shows the zoomed in regions. Four markers on these images were selected exactly at the same place in the third row to compare the intensity and velocity profiles of the SR images. Polynomial fitted profiles to the pixel values of the SR images quantify diameter and flow velocity of the selected vessels in the bottom row. 

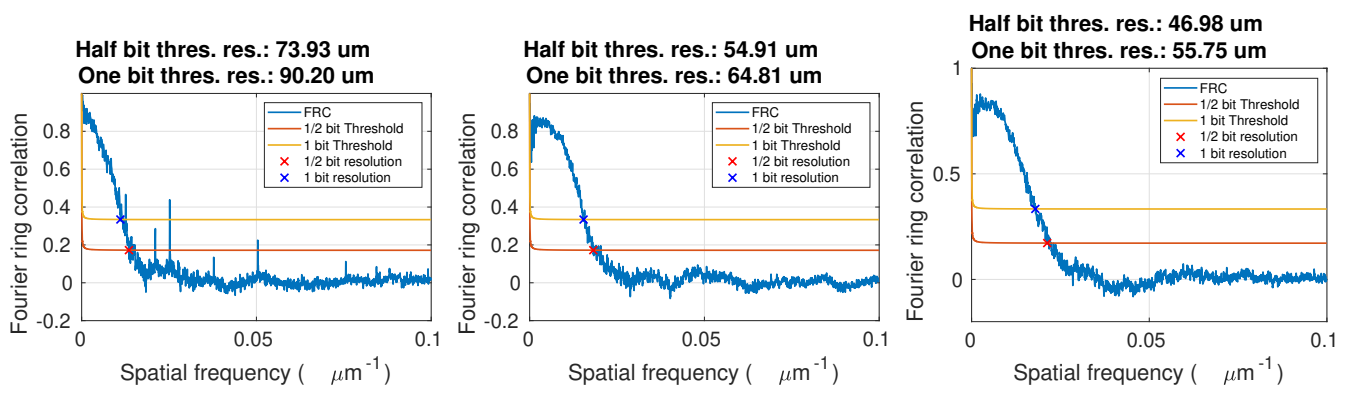

Fig. 14. FRC curves and bit-based information threshold for SR images (a) without motion correction (b) with local motion estimation and global correction (c) with motion-field estimation and local correction.

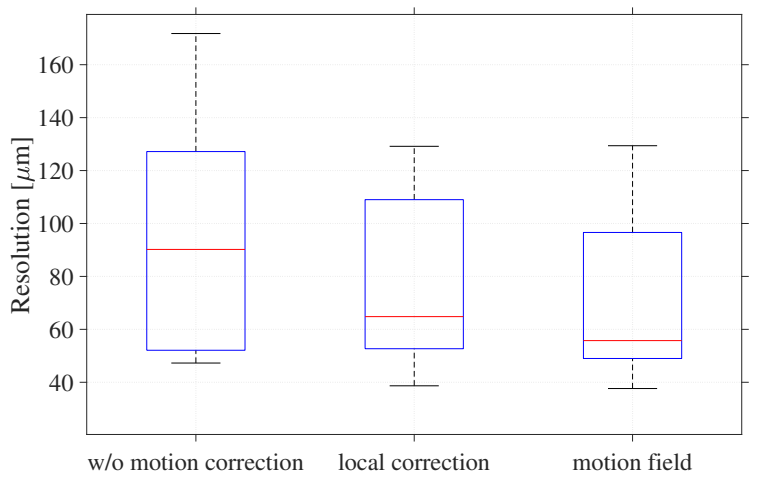

Fig. 15. Box plots of FRC metric with one-bit threshold for 3 methods and 10 SR images of different kidneys.

velocity of roughly $6 \mathrm{~mm} / \mathrm{s}$. The two last profiles showed how having MB tracks, which are not compensated for the motion appropriately, could lead to unusually narrow or wide vessels.

Estimation of microvascular blood flow deep inside organs is difficult in vivo. Older studies using invasive video microscopy techniques have estimated the mean red blood cell velocity in the descending (arterial) vasa recta at the tip of the renal papilla (at the very bottom of the medulla) of rat kidneys to be $\approx 1 \mathrm{~mm} / \mathrm{s}$ [36-38]. This is in the range of the velocity profile 3 from the medulla shown in Fig. 13. The remaining velocity profiles include a mixture of differently sized arcuate and cortical radial arteries and veins. Validation of the velocities in the cortex will require separation and categorization of the different types of vessels. For larger vessels, spectral Doppler may help validate the SRI-derived velocity estimations. For the cortical microvasculature validation is very challenging, as no other techniques are available to make it noninvasively and in vivo. Different video microscopy approaches have also been used in the cortex. One study in vitro blood perfused kidneys from rats found a velocity of $\approx 10 \mathrm{~mm} / \mathrm{sec}$ in the afferent arterioles [39]. Another study estimated blood flow in different renal arteries and arterioles of varying size in a split hydronephrotic kidney and reported velocities ranging from $0.5-65 \mathrm{~mm} / \mathrm{s}$ but did not describe how the velocities related to vessel size [40]. Therefore, if SRI can provide precise estimations of blood flow velocity in different vascular segments of the kidney, it will fulfill an unmet need and allow evaluation of intrarenal blood flow in various physiological and pathological states.
The FRC resolution metric is shown in Fig. 15. The metric is controversial because of its dependency on splitting the SR image, threshold criteria, and pixel values (or the number of scatterers) in each image. However, since the images from 10 rat kidneys were split, scaled, and thresholded the same way, the metric is still helpful for quality assessment even though they may not represent the actual resolution. The metric showed a large improvement in introducing the local motion correction and a less dramatic improvement, when employing the global motion field.

The approach assumes that the out-of-plane motion is negligible. The probe used has an elevation F-number of nearly 6 at $20 \mathrm{~mm}$ giving that the elevation slice thickness is at least $6 \lambda=1.2 \mathrm{~mm}$, which is well above the estimated peak axial and lateral motion of $100-200 \mu \mathrm{m}$. The change in amplitude of motion in and out of the imaging plane will therefore be negligible and not affect detection of the bubbles. Also note that the detection of centroid removes the amplitude of the bubble, so the variation in amplitude will not be seen by the tracking algorithm. Attaining full super resolution in the elevation direction will necessitate a full 3-D measurements and must employ either sparse 2-D matrix probes [41] or rowcolumn probes [42, 43].

A few rat experiments showed a decreased precision of up to $20 \mu \mathrm{m}$. This was due to parts of the estimated motion data being out of the search range precluding motion correction. This was found in three of the early rat experiments and might have been from accidentally moving parts of the experiments, and was only seen in experiment 1,2 , and 5. Excluding these always yields a precision below $10 \mu \mathrm{m}$ and often much lower, indicating that this set-up and processing pipeline has the potential of capillary flow visualization.

\section{CONCLUSION}

A motion correction super resolution pipeline has been presented and used on in vivo data from the left kidney of Sprague-Dawley rats. It was shown that the tissue motion can be reliably determined with a precision in the $\mu \mathrm{m}$ range, and that is can be used for compensating for the motion from breathing, heartbeating, and muscle contractions.

\section{ACKNOWLEDGMENT}

This work was financially supported by grant 82-2014-4 from the Danish National Advanced Technology Foundation, 
by grant 7050-00004B from Innovation Fund Denmark, and from BK Medical, Herlev, Denmark.

\section{REFERENCES}

[1] O. M. Viessmann, R. J. Eckersley, K. ChristensenJeffries, M. X. Tang, and C. Dunsby, "Acoustic superresolution with ultrasound and microbubbles," Phys. Med. Biol., vol. 58, pp. 6447-6458, 2013.

[2] M. A. O'Reilly and K. Hynynen, "A super-resolution ultrasound method for brain vascular mapping," Med. Phys., vol. 40, no. 11, pp. 110701-7, 2013.

[3] Y. Desailly, J. Pierre, O. Couture, and M. Tanter, "Resolution limits of ultrafast ultrasound localization microscopy," Phys. Med. Biol., vol. 60, no. 22, pp. 8723 8740, 2015.

[4] K. Christensen-Jeffries, R. J. Browning, M. Tang, C. Dunsby, and R. J. Eckersley, "In vivo acoustic superresolution and super-resolved velocity mapping using microbubbles," IEEE Trans. Med. Imag., vol. 34, no. 2, pp. 433-440, February 2015.

[5] C. Errico, J. Pierre, S. Pezet, Y. Desailly, Z. Lenkei, O. Couture, and M. Tanter, "Ultrafast ultrasound localization microscopy for deep super-resolution vascular imaging," Nature, vol. 527, pp. 499-502, November 2015.

[6] D. Ackermann and G. Schmitz, "Detection and tracking of multiple microbubbles in ultrasound B-mode images," IEEE Trans. Ultrason., Ferroelec., Freq. Contr., vol. 63, no. 1, pp. 72-82, January 2016.

[7] K. Christensen-Jeffries, O. Couture, P. A. Dayton, Y. C. Eldar, K. Hynynen, F. Kiessling, M. O’Reilly, G. F. Pinton, G. Schmitz, M. Tang et al., "Super-resolution ultrasound imaging," Ultrasound Med. Biol., vol. 46, no. 4, pp. 865-891, 2020.

[8] S. Harput, K. Christensen-Jeffries, J. Brown, Y. Li, K. J. Williams, A. H. Davies, R. J. Eckersley, C. Dunsby, and M. Tang, "Two-stage motion correction for superresolution ultrasound imaging in human lower limb," IEEE Trans. Ultrason., Ferroelec., Freq. Contr., vol. 65, no. 5, pp. 803-814, 2018.

[9] K. B. Hansen, C. A. Villagomez-Hoyos, J. Brasen, K. Diamantis, V. Sboros, C. M. Sørensen, and J. A. Jensen, "Robust microbubble tracking for super resolution imaging in ultrasound," in Proc. IEEE Ultrason. Symp., 2016, pp. 1-4.

[10] J. Foiret, H. Zhang, T. Ilovitsh, L. Mahakian, S. Tam, and K. W. Ferrara, "Ultrasound localization microscopy to image and assess microvasculature in a rat kidney," Scientific Reports, vol. 7, no. 1, pp. 13 662:1-12, 2017.

[11] V. Hingot, C. Errico, M. Tanter, and O. Couture, "Subwavelength motion-correction for ultrafast ultrasound localization microscopy," Ultrasonics, vol. 77, pp. 17-21, 2017.

[12] S. Harput, M. Toulemonde, A. Ramalli, K. ChristensenJeffries, E. Boni, P. Tortoli, C. Dunsby, and M. Tang, "Quantitative microvessel analysis with 3-D superresolution ultrasound and velocity mapping," in Proc. IEEE Ultrason. Symp., 2020, pp. 1-4.
[13] M. Piepenbrock, S. Dencks, and G. Schmitz, "Reliable motion estimation in super-resolution us by reducing the interference of microbubble movement," Proc. IEEE Ultrason. Symp., vol. 2019, p. 8925566, 2019.

[14] T. M. Kierski, D. Espindola, I. G. Newsome, E. Cherin, J. Yin, F. S. Foster, C. E. M. Demore, G. F. Pinton, and P. A. Dayton, "Superharmonic ultrasound for motionindependent localization microscopy: Applications to microvascular imaging from low to high flow rates," IEEE Trans. Ultrason., Ferroelec., Freq. Contr., vol. 67, no. 5, pp. 957-967, 2020.

[15] J. A. Jensen, S. B. Andersen, C. A. V. Hoyos, K. L. Hansen, C. M. Sørensen, and M. B. Nielsen, "Tissue motion estimation and correction in super resolution imaging," in Proc. IEEE Ultrason. Symp., 2019, pp. 1-4.

[16] R. J. Eckersley, C. T. Chin, and P. N. Burns, "Optimising phase and amplitude modulation schemes for imaging microbubble contrast agents at low acoustic power," Ultrasound Med. Biol., vol. 31, no. 2, pp. 213-219, 2005.

[17] V. Hingot, C. Errico, B. Heiles, L. Rahal, M. Tanter, and O. Couture, "Microvascular flow dictates the compromise between spatial resolution and acquisition time in ultrasound localization microscopy," Scientific Reports, vol. 9, no. 1, pp. 1-10, 2019.

[18] T. Furnival, R. K. Leary, and P. A. Midgley, "Denoising time-resolved microscopy image sequences with singular value thresholding," Ultramicroscopy, vol. 178, pp. 112 124, 2017.

[19] U. W. Lok, P. Song, J. D. Trzasko, R. Daigle, E. A. Borisch, C. Huang, P. Gong, S. Tang, W. Ling, and S. Chen, "Real time SVD-based clutter filtering using randomized singular value decomposition and spatial downsampling for micro-vessel imaging on a Verasonics ultrasound system," Ultrasonics, vol. 107, p. 106163 , 2020.

[20] M. K. Cheezum, W. F. Walker, and W. H. Guilford, "Quantitative comparison of algorithms for tracking single fluorescent particles," Biophys. J., vol. 81, no. 4, pp. 2378-2388, 2001.

[21] I. N. Bankman, Handbook of Medical Image Processing and Analysis. Elsevier Inc., 2009.

[22] K. G. Brown, D. Ghosh, and K. Hoyt, "Deep learning of spatiotemporal filtering for fast super-resolution ultrasound imaging," IEEE Trans. Ultrason., Ferroelec., Freq. Contr., vol. PP, no. 99, pp. 1-1, 2020.

[23] X. Liu, T. Zhou, M. Lu, Y. Yang, Q. He, and J. Luo, "Deep learning for ultrasound localization microscopy," IEEE Trans. Med. Imag., vol. 39, no. 10, pp. 3064-3078, 2020.

[24] G. E. Trahey, J. W. Allison, and O. T. von Ramm, "Angle independent ultrasonic detection of blood flow," IEEE Trans. Biomed. Eng., vol. BME-34, no. 12, pp. 965-967, 1987.

[25] X. Wu, "An efficient antialiasing technique," Computer Graphics, vol. 25, no. 4, pp. 143-152, 1991.

[26] R. P. Nieuwenhuizen, K. A. Lidke, M. Bates, D. L. Puig, D. Grunwald, S. Stallinga, and B. Rieger, "Measuring image resolution in optical nanoscopy," Nat. Methods, 
vol. 10, no. 6, pp. 557-562, 2013.

[27] N. Banterle, K. H. Bui, E. A. Lemke, and M. Beck, "Fourier ring correlation as a resolution criterion for super-resolution microscopy," J. Struct. Biol, vol. 183, no. 3, pp. 363-367, 2013.

[28] M. van Heel and M. Schatz, "Fourier shell correlation threshold criteria," J. Struct. Biol, vol. 151, no. 3, pp. 250-262, 2005.

[29] J. A. Jensen and N. B. Svendsen, "Calculation of pressure fields from arbitrarily shaped, apodized, and excited ultrasound transducers," IEEE Trans. Ultrason., Ferroelec., Freq. Contr., vol. 39, no. 2, pp. 262-267, 1992.

[30] J. A. Jensen, "Field: A program for simulating ultrasound systems," Med. Biol. Eng. Comp., vol. 10th Nordic-Baltic Conference on Biomedical Imaging, Vol. 4, Supplement 1, Part 1, pp. 351-353, 1996.

[31] — "A multi-threaded version of Field II," in Proc. IEEE Ultrason. Symp., 2014, pp. 2229-2232.

[32] S. B. Andersen, I. Taghavi, C. A. V. Hoyos, S. B. Søgaard, F. Gran, L. Lonn, K. L. Hansen, J. A. Jensen, M. B. Nielsen, and C. M. Sørensen, "Super-resolution imaging with ultrasound for visualization of the renal microvasculature in rats before and after renal ischemia: A pilot study," Diagnostics, vol. 10, no. 11, p. 862, 2020.

[33] J. A. Jensen, S. Nikolov, K. L. Gammelmark, and M. H. Pedersen, "Synthetic aperture ultrasound imaging," $\mathrm{Ul}$ trasonics, vol. 44, pp. e5-e15, 2006.

[34] M. Tanter and M. Fink, "Ultrafast imaging in biomedical ultrasound," IEEE Trans. Ultrason., Ferroelec., Freq. Contr., vol. 61, no. 1, pp. 102-119, January 2014.

[35] S. Dencks, M. Piepenbrock, and G. Schmitz, "Assessing vessel reconstruction in ultrasound localization microscopy by maximum likelihood estimation of a zeroinflated poisson model," IEEE Trans. Ultrason., Ferroelec., Freq. Contr., vol. 67, no. 8, p. 9032203, 2020.

[36] C. Holliger, K. V. Lemley, S. L. Schmitt, F. C. Thomas, C. R. Robertson, and R. L. Jamison, "Direct determination of vasa recta blood flow in the rat renal papilla." Circ. Research, vol. 53, no. 3, pp. 401-413, 1983.

[37] B. Zimmerhackl, C. R. Robertson, and R. L. Jamison, "Fluid uptake in the renal papilla by vasa recta estimated by two methods simultaneously," Am. J. Physio., vol. 248, no. 3, pp. F347-53, 1985.

[38] W. A. Cupples, T. Sakai, and D. J. Marsh, "Angiotensin ii and prostaglandins in control of vasa recta blood flow," Am.J. Phys.-renal Phys., vol. 254, no. 3, pp. F417-F424, 1988.

[39] T. Takenaka, L. M. Harrison-Bernard, E. W. Inscho, P. K. Carmines, and L. G. Navar, "Autoregulation of afferent arteriolar blood flow in juxtamedullary nephrons," Am. J. Physio., vol. 267, no. 5, pp. F879-87, 1994.

[40] A. S. D. Vriese, T. J. Verbeuren, M. O. Vallez, N. H. Lameire, M. D. Buyzere, and P. M. Vanhoutte, "Off-line analysis of red blood cell velocity in renal arterioles," $J$. Vasc. Research, vol. 37, no. 1, pp. 26-31, 2000.

[41] S. Harput, K. Christensen-Jeffries, A. Ramalli, J. Brown, J. Zhu, G. Zhang, C. H. Leow, M. Toulemonde, E. Boni, P. Tortoli, R. J. Eckersley, C. Dunsby, and M. Tang, “3-D super-resolution ultrasound (SR-US) imaging with a 2-D sparse array," arXiv preprint, p. 1902.01608v1, 2019.

[42] J. A. Jensen, M. Schou, M. L. Ommen, S. H. Øygard, T. Sams, M. B. Stuart, E. V. Thomsen, N. B. Larsen, C. Beers, and B. G. Tomov, "3-D super resolution imaging using a $62+62$ elements row-column array," in Proc. IEEE Ultrason. Symp., 2019, pp. 1-4.

[43] J. A. Jensen, M. L. Ommen, S. H. Øygard, M. Schou, T. Sams, M. B. Stuart, C. Beers, E. V. Thomsen, N. B. Larsen, and B. G. Tomov, "Three-dimensional super resolution imaging using a row-column array," IEEE Trans. Ultrason., Ferroelec., Freq. Contr., vol. 67, no. 3, pp. 538-546, 2020.

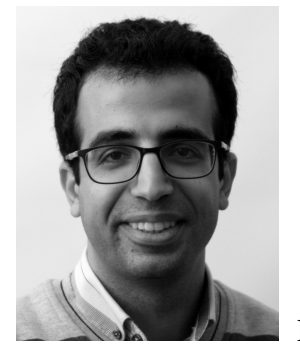

Iman Taghavi received the B.Sc. and M.Sc. degrees both in electrical engineering from the University of Isfahan, Iran, in 2013 and 2016. From September 2013 to June 2015, he was also a research assistant at the Isfahan University advanced communications laboratory. He is currently a Ph.D. fellow student at the Center for Fast Ultrasound Imaging at the Technical University of Denmark. His research interests include signal processing and its applications in array sensors, radars, and biomedical imaging.

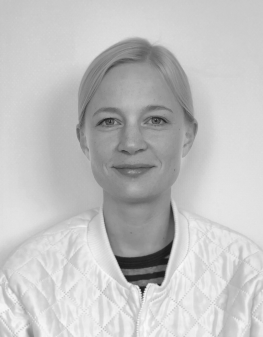

Sofie Bech Andersen received a master's degree in medicine from the University of Copenhagen, Denmark, in 2015. After concluding a year as a resident (introductory position) in radiology at Rigshospitalet in Copenhagen, she began and is still currently doing a Ph.D. in pre-clinical ultrasound super-resolution imaging in a collaboration between the Department of Biomedical Sciences at the University of Copenhagen, the Department of Radiology at Rigshospitalet, the Center for Fast Ultrasound Imaging at the Technical University of Denmark, and BK Medical. Her research interests include the development of diagnostic imaging tools for use in the clinic.

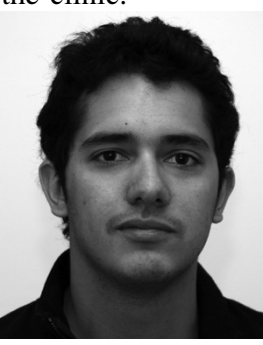

Carlos Armando Villagómez Hoyos was born in 1985. He received the B.Sc. degree in electronics engineering and the M.Sc. degree in digital signal processing from the National Autonomous University of Mexico, Coyoacán, Mexico, in 2008 and 2013, respectively. He received his Ph.D. degree in biomedical engineering with the Center for Fast Ultrasound Imaging, Technical University of Denmark, Kongens Lyngby, Denmark, with research on optimal synthetic aperture duplex imaging. He is currently employed 
at BK Medical as a System engineer.

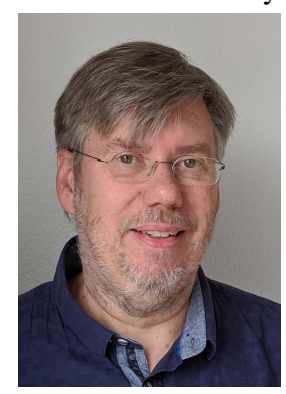

Michael Bachmann Nielsen is a Medica graduate from the Faculty of Health Science, University of Copenhagen, Copenhagen, Denmark, in 1985, and received the Ph.D. degree in 1994, and the Dr.Med. Dissertation degree in 1998. He is a Full Professor of Oncoradiology with the University of Copenhagen, and a Consultant with the Department of Radiology, Rigshospitalet, Copenhagen. He has authored over 250 peer-reviewed journal articles on ultrasound or radiology. His current research interests include clinical testing of new ultrasound techniques, tumor vascularity, ultrasound elastography, training in ultrasound and artificial intelligence.

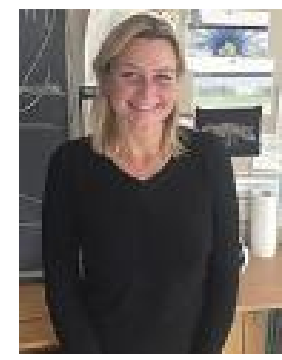

Charlotte Mehlin Sørensen has a B.Sc. in Biology; an M.Sc in Human Biology and a Ph.D in renal physiology from University of Copenhagen. After obtaining the $\mathrm{PhD}$ degree, she was employed as Assistant Professor at Department of Biomedical Sciences. During one year leave of absence she worked as a drug discovery project leader at Zealand Pharma but came back to continue as Associate Professor. Her research focuses on renal hemodynamics, diabetes and hypertension. Changes in renal autoregulation and blood flow regulation as a consequence of changes in the topology of the renal vascular tree, intercellular communication and ion channel expression is investigated in vivo in different disease models. She runs a state-of-the-art in vivo rodent laboratory for acute and chronic experimental set-ups. She is a member of American Physiological Society, Danish Hypertension Society the Scandinavian Physiological Society and Danish Cardiovascular Academy. She has published 52 peer-reviewed papers in international journals.

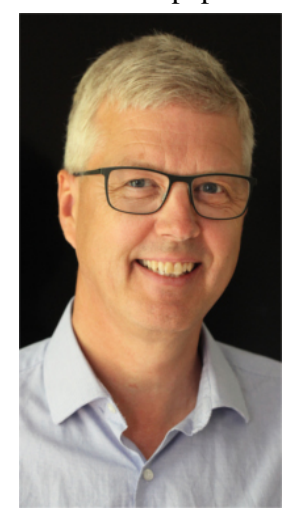

Jørgen Arendt Jensen (M'93-SM'02-F'12) received the MSc degree in 1985, the Ph.D. degree in 1989, and the Dr.Techn. degree all from the university in 1996. Since 1993, he has been a Full Professor of Biomedical Signal Processing with the Department of Health Technology, Technical University of Denmark. He has been the founder and head of the Center for Fast Ultrasound Imaging since its inauguration in 1998. CFU has contributed with innovations in transverse oscillation vector flow imaging, synthetic aperture flow imaging in 2-D and 3-D, ultrasound simulation, research scanners, and row-column probes and beamforming. He has published more than 500 journal and conference papers on signal processing and medical ultrasound and the book Estimation of Blood Velocities Using Ultrasound (Cambridge Univ. Press), 1996. He is also the developer and maintainer of the Field II simulation program. He has been a visiting scientist at Duke University, Stanford University, and the University of Illinois at Urbana-Champaign. He was founder and head of the Biomedical Engineering group from 2007 to 2010. In 2003, he was one of the founders of the biomedical engineering program in Medicine and Technology, which is a joint degree program between the Technical University of Denmark and the Faculty of Health and Medical Sciences at the University of Copenhagen. The degree is one of the most sought-after engineering degrees in Denmark. He was chairman of the study board from 2003 to 2010 and Adjunct Professor with the University of Copenhagen from 2005 to 2010 . He has given a number of short courses on simulation, synthetic aperture imaging, and flow estimation at international scientific conferences and teaches biomedical signal processing and medical imaging at the Technical University of Denmark. His research is centered around simulation of ultrasound imaging, synthetic aperture imaging, vector blood flow estimation, 3-D and super resolution imaging, row-column probes, and construction of ultrasound research systems. He has educated $45 \mathrm{PhD}$ students and currently advises $18 \mathrm{PhD}$ students. Dr. Jensen has given more than 60 invited talks at international meetings and received several awards for his research, most recently the Grand Solutions Prize from the Danish Minister of Science, the order of the Dannebrog by her Majesty the Queen of Denmark, and the Rayleigh award from the UFFC Society in the field of Ultrasonics in 2019. 